\title{
Pediatric Obesity and Bariatric Surgery
}

\author{
Participants:
}

\author{
Lisa Rowen, DNSc, RN, FAAN \\ Editor-in-Chief \\ Journal of Bariatric Nursing and Surgical \\ Patient Care
}

\author{
Meg Zeller, PhD \\ Associate Professor of Pediatrics \\ Cincinnati Children's Hospital \\ Medical Center \\ Cincinnati, $\mathrm{OH}$
}

Lori Mooney, RD, LD

Center for Healthy Weight and Nutrition

Nationwide Children's Hospital

Columbus, $\mathrm{OH}$

\author{
Renay D. Tyler, MSN, ACNP, CNSN \\ Associate Editor, Series Co-Editor \\ Journal of Bariatric Nursing and Surgical \\ Patient Care
}

\author{
Stephenie Wallace, MD, MSPH \\ Assistant Professor \\ Medical Director, \\ Center for Weight Management The Children's \\ Hospital of Alabama \\ Birmingham, $A L$
}

\author{
Beverly Haynes, RN, CPN \\ Bariatric Program Patient Care Coordinator \\ Children's Health System \\ Birmingham, $A L$
}

T

HIS ISsue's Roundtable Discussion addresses childhood and adolescent obesity. A growing focus and attention have been placed on childhood and adolescent obesity over the past year. In part, this increased attention has been spearheaded by Michele Obama and her campaign to make the nation's children healthier. The national goal is to solve the childhood obesity epidemic within a generation and involves an integrated strategy that includes physical activity, making healthy and affordable food available, and education for parents and children. To learn more about the plan and its 70 specific strategies, access http://www.letsmove.gov/taskforce_ childhoodobesityrpt.html for details.

\section{- Lisa Rowen, DNSc, RN, FAAN Editor-in-Chief}

Lisa Rowen: I would like to begin by asking each of you if you believe that the obesity epidemic among adolescents is improving, staying the same, or getting worse, and why do you think that?
Stephenie Wallace: I believe the obesity epidemic in the adolescent population is still in a stage where it is getting worse. As an example, I am seeing more teens on atypical antipsychotic medications which can cause changes in glucose metabolism, weight gain, and dyslipidemia. Recently, I was impressed by an article in the Archives of Pediatrics and Adolescent Medicine talking about psychiatric medications and how we, pediatricians, are not properly screening our teens on these medications.

Lori Mooney: I think what we have seen is a slight leveling off within some of the age groups, but the trajectory is still going strong in others. It is certainly quite prevalent in the Hispanic and African American populations.

Beverly Haynes: I have to agree with Stephenie in that we are not seeing, at least where we are located, much leveling off or even a decrease right now. We may even be seeing more of these kids coming through our system, but according to the Centers for Disease Control 
and Prevention (CDC), we are supposed to be leveling off at this time.

Lori Mooney: That is what they are saying regarding the older children, correct?

Beverly Haynes: For children and adolescents. The exception is supposed to be the group which includes 6- to 19-year-old boys. However, at our site, we are not recognizing any leveling off.

Meg Zeller: I think you are correct. From the national standpoint, it is stabilizing in terms of prevalence. However, I think it is alarming and pertinent to this roundtable that the Body Mass Index (BMI) is shifting to the right, so that we have more young people who are severely obese or extremely obese, greater than the 99th percentile. When we talk about prevalence and we talk about a leveling off, there is this feeling of "Oh, good, the prevalence is not increasing." But what gets lost is that we also have bigger kids, which is not decreasing, and, due to this, more kids who will probably be in great need of effective intervention.

Lori Mooney: I think since the adolescent obesity issue is such a multifactorial disease, with several contributing factors, we are looking at genes, environmental and socioeconomic factors, as well as the energy imbalance issue. I would agree that we are seeing more severely obese patients with increased morbidity issues who are certainly at risk, whether it is hypertension, orthopaedic issues, metabolic syndrome, non-alcoholic steatohepatitis, or obstructive sleep apnea. It is there, and certainly with type 2 diabetes, we are seeing it in very young children. We are now seeing so many comorbid issues in obese kids that we did not see before.

Lisa Rowen: To follow that, when an adolescent with extreme obesity presents to you, what clinical, psychological, and emotional indicators are you observing, typically?

Stephenie Wallace: From a clinical standpoint, I am seeing a lot of irregular menses and hirsutism that suggest polycystic ovarian syndrome (PCOS). I follow the most recent guidelines recommending that everybody have fasting lipid level, and fasting glucose, liver function test to screen for diabetes, nonalcoholic steatohepatitis, and dyslipidemia. I am seeing a lot of elevated BP [blood pressure] concerning for hypertension. I do try to follow them closely for two or three visits before I decide to treat. I do a lot of counseling with them on salt-containing foods, and, of course, work on weight loss.

Before I consider particularly bariatric surgery, I first want to identify the family's objective. How much weight is it that they believe should be lost and why? What are their goals? Is it for their health? Is it social? I find it usually is a combination of both when I query the family and the adolescent together and in front of me. Then, how "aggressive" do they want to be? There are a lot of young people that we follow in our center who I believe have medical indicators for bariatric surgery, but the procedure and the risks involved suggest this surgery is not right for them.

If these adolescents want to try to lose weight clinically, I am more than happy to follow them as close as their schedule and my schedule will allow, and see if we can get the weight off medically. However, if their health is getting worse, then I will revisit the topic of bariatric surgery as a way of possibly improving their quality of health and life.

Lori Mooney: That was said very well. I think the real objective is that we try to do something with a weight management approach as much as possible. We are all becoming much better at directing these patients directly into a bariatric program if that presents itself for evaluation.

Lisa Rowen: I would like to hear your thoughts on the best prevention and treatment methods for adolescents with extreme obesity. You talked about weight management. What would a holistic approach look like?

Lori Mooney: Speaking from the nutritional side, it is important to involve the family in changes that can be made in the home. I am going to go back to the guidelines that were established by the Academy of Pediatrics in 2007, when we had the expert committee recommendations regarding prevention and assessment of kids with obesity issues. That 
summary was a wonderful way of identifying several areas that we could address immediately.

Sitting down with the family is very important. The positive nutritional aspects from having lean proteins and more fruits and vegetables at each meal, rather than heavy starch portions, are critical to long-term success. Many of our kids are getting far too many of these starches on a regular basis.

We assume that people know about proper nutrition and healthy eating habits. However, it has become very obvious to me that a lot of people do not know enough about these very basic issues. And it is important to tackle them: eating healthy, eating less fast food, portion sizes, and eating more meals at home. Additionally, sweetened beverages are a considerable issue for our children. They are taking in so many more empty calories than with the smaller size beverages of 20 years ago. It is important to take a look at this whole distortion in portions and the ingredients in beverages.

We also need to look at entertainment in the home, limiting TV, computer, and video game time to no more than 2 hours a day.

Getting adolescents to eat breakfast every day is another area that needs addressing. We all know the importance of starting the day with a good breakfast. If this does not happen, then children tend to eat more later on in the day and consume larger quantities of food. So these are some of the issues that we tackle and need to continue doing so effectively.

Stephenie Wallace: I agree. When I am wearing my primary care hat, I usually try to pick one or two of those areas and really create a focus using an in-depth conversation, typically with the young person that I am counseling. We also have been paying a lot of attention to smoking or stop smoking, to be very holistic as another factor that may be contributing to weight gain. Also emotional eating.... Is their depression adequately being treated and managed? When are they binging? I know that Heather Austin, PhD, our clinic coordinator, pediatric psychologist, and our colleague, spends a lot of time with our teens helping with that. And as I mentioned before I have started paying attention to some of the other medications that may be contributing to weight gain, namely, the psychiatric medications. Weight gain is one of the side effects, and our families need to combat the side effects with behavioral changes for a healthy lifestyle.

Then, lastly, stressing to the families that their children and their teens are going to do what they see them do. If the parents are not exercising, then the teens are not going to exercise, and it is not fair for you [the parent] to sit down and ask the children to run all over the place. As a leader of your home, as the parent of the house, be a parent, set limits, set goals, set standards, and ask your families to follow them and give consequences when they are not reached.

Lori Mooney: Absolutely, those are all very, very important models. I want to point out a few more nutritional issues, and then mention how important physical activity is for these families. Getting a diet rich in calcium is crucial. We are seeing a lot more sweetened beverage consumption and a lot less milk and dairy intake, so we want to enhance and encourage the latter.

We really want the children to be eating diets high in fiber, so getting them to understand what that means and instigating small changes, such as replacing white rice with brown rice and eating $100 \%$ whole wheat bread, are a big deal for some families, and not always easily done.

The other problem is getting rid of these energy-dense foods and integrating more nutrient-dense foods into family meals, foods that have lots of vitamins and mineral components to them, as well as protein, rather than the nutrient deficiency of typical snacking foods. We are just doing way too much "snacking" in this country. I think kids are beginning to eat their meals in multiple snacks rather than sitting down and eating three meals a day and maybe a healthy snack later on. Eliminating or at least controlling the amount of energy-dense foods consumed is a real and significant challenge.

Meg Zeller: I agree with all the points being said, and I am trying to come up with new points to be made. I think we are hinting toward family change, as well as what is prominent for an adolescent, whether it is someone seeking bariatric surgery or a more traditional 
behavioral weight management. It is important to take on the fact that among the people sitting in the room with you, be it the parents or siblings or whoever comprises the family unit, nine times out of ten, there is going to be another obese person in the room. We are finding this nationally, and if you just think about your clinics, it is being mirrored there as well. You will probably agree that there is an extremely obese family member in the clinic room with you or one at home who does not come in, and that the obesity that has moved to extreme levels did not occur overnight.

When "obesity" has been happening for a long time in that family, we need to come up with ways that are family friendly enough and that are not threatening but realistic for the family to make significant changes in how food works in the household. A teen is not going to be successful in their weight loss, be it through surgical weight loss or behavioral weight loss or some type of medication, if the family unit is not changing how it approaches food. Next, the adolescent needs to work on changing how he or she takes control of that and their own food choices because they eat a lot of their meals outside of the home and at school.

I think we do not talk enough about adolescent obesity and the home environment. We talk about what the family should do, but we do not talk about how we are going to get them to do it and the clinical practice of how that gets done.

Our other colleagues, such as Kevin Smith, $\mathrm{PhD}$, at Nationwide Children's Hospital and Heather, whom I mentioned prior, and myself are asked all the time, "How do we assess a family to decide whether a family member is appropriate for bariatric surgery?" We wish we had the answer to that question. Traditional methods of assessing a family with a questionnaire are not hitting "the nail on a head" and giving us some answer that spits out and says to us, "No, that family is not appropriate." We do not know yet, but the writing is on the wall, I think very obviously that if a family is sitting there with more than one person who is extremely obese, we need to take a different approach than just treating the adolescent.

Lisa Rowen: I would like to follow up on that issue and ask all of you if you have found any family strategies that have been particularly effective, ones that you have been able to offer families. As well, have you seen actual outcomes and effectiveness through particular strategies?

Lori Mooney: The recommendations continue to look at very specific eating behaviors as well as physical activity, focusing on getting rid of the sedentary behaviors that these families tend to adopt. It is not one without the other. It has to be both.

And these healthy habits take time. The issue is that it is very difficult to make behaviorbased weight loss changes. Given the fact that these young people come to us already overweight, the target of the obesity prevention needs to start at the early child state, beginning at birth and continuing with lifestyle behaviors to prevent the obesity, rather than improve it at the other end once obesity is there.

That being said, we are still looking at limiting sugary beverages, encouraging the consumption of diets that have increased amounts of fruits and vegetables, restricting television, eating breakfast daily, and reducing the number of restaurant visits. We are going to do away with many existing unhealthy temptations and opportunities, which is why limiting them is crucial. For example, frequently eating fast food is one cause of obesity, so we need to look at the healthier options, such as encouraging family meals, because we know through research that family meals are associated with higher quality diets in general. Limiting portion size is the other real issue. Therefore, the nutrition side continues to be where the evidence supports change. We have got to target behaviors.

Meg Zeller: A vital question is "How do we do that at a family level?" I am curious what you think. Listing all those things makes a lot of sense to me, and I agree with everything you are saying. However, how do you clinically make that happen?

Stephenie Wallace: I cannot really say from a public health standpoint what one intervention works for every family. I have been impressed with several of our families and how they have taken our advice given to them in some of our clinic visits and adapted it for their home. What 
has definitely worked is when the parents decide, "I am going to do this with my child," or "I want to lose weight for my health." When the parents say, "Let me change, and let everybody in the house change too," then I tend to see results and the teen loses weight.

An example is from a family in our clinic who changed the Epstein Stoplight diet to fit them. We teach this plan to our patients and families, and common foods are rated red, yellow, and green, like a stop light, according to calorie and fat content. Our nutritionists will go over this program with the families in our clinic; it is well researched and well promoted for pediatric obesity treatment. Our program is a modified Epstein plan where we recommend one stop food per day. However, one of our families decided that they will avoid all stop food during the month but would have one day a month where they were not as vigilant. That is, they would save their favorite sweets for that day. Talking to them, I ask them very carefully, "Are you guys overeating the sweets on that day?" They would say, "No, I tend to eat less than I was before. I just save my one piece of chocolate cake for that special day each month."

But this strategy was adopted from the top down with the parents participating too. There was a friendly competition between the parents and the children on who could stick to the plan and only avoid the high-calorie foods. When I saw the patient back for one of their visits, he had lost weight, and I was really pleased.

I also encourage those families who have the resources to do added things, programs such as Weight Watchers or a gym, and to use some of the online weight loss programs. I think if there is more support, whether it is online or in person, it is valuable. I have seen some results for some of our older teens in Curves or in Weight Watchers or with Jenny Craig. When they come in to see us, our nutritionists make sure that they are getting sufficient calcium and iron, those things that some of our adult programs tend to not stress as much with teenagers. But our nutritionists are very pleased that they are receiving those necessary vitamins and minerals and other nutrients and having success at weight loss.

Lori Mooney: I think the behavior change is effective only if the parent or family recog- nizes the problem and wants to address it. If they are not ready for change, it is very difficult for any change to take place. Part of our role is to help families identify where their problems lie and one or two things that they can work on between now and the next time we see them, in order to encourage obesity prevention-type behaviors or changes in current behaviors.

It is a lot of work. It is exhausting work. It is very time-consuming. That being said, on the nutrition side of things, it can be as simple as just getting them to fill half of their plate with fruits and vegetables, a simple concept of rearranging the plate, and using a smaller plate. There are lots of little techniques you can do behind the scenes.

Hopefully, when you see families getting more fruits and vegetables it is displacing the amount of very starchy foods that they were eating or the overabundance of protein items they may be placing on their plate. It is a combination of really trying to educate and reeducate, and constantly trying to tackle simple things and changes that these families can be effective in making and experience small successes.

Lisa Rowen: We have talked a lot about the families. What about the government? What type of government intervention or support could be helpful to support the needs of the extremely obese adolescent population? We have heard a lot from Michelle Obama and her agenda for having the youth of the country "move," to stay very aware of what children are consuming, and to look at what we are doing in schools regarding obesity and food issues. What could be done? Are there any particular government interventions or support that you believe would be helpful?

Lori Mooney: Certainly, public policy is part of this obesity issue. It is not just one thing that has happened; it is multifaceted.

Beverly Haynes: I remember when I was in school that physical education, or $P E$ as we called it, was mandatory. I do not think it is now, because I know my children have not been in a mandatory situation at school regarding exercise activities, and I have read with horror that recess has been eliminated in a lot 
of elementary schools. I am just concerned that we are overlooking those key ways to get some exercise in with these kids, and in so doing are saying that it is not important in our school systems. If we have to have some further government influence, that is okay with me; it does not have to be just academics all the time; we can get some exercise in as well. It goes back to the old saying, "All work and no play makes Jack a dull boy." I think the obesity epidemic is proof of this.

Stephenie Wallace: I would add that I think where government can be helpful is giving our schools money for the school lunches. Even simple changes, such as making accommodations for teens with diabetes or serving fewer breaded products, could make a difference. However, particularly in Alabama, when you get into some of our very small school systems, those school systems are struggling, and it is very hard to make even some of these simple changes that might help against obesity.

I enjoyed watching a couple of episodes of Jamie Oliver's Food Revolution. I think it was interesting to see the response of the community, how he showed the community what some of the school lunches are made of, and how these foods affect our body. I feel for our school systems. They just do not have the money with everything else that we parents are asking them to do to educate our children. So it is hard for the schools to make some of the changes regarding activity and nutrition that we think would be helpful in this area. But for a lot of kids, the schools have a huge role in what they eat. Many kids coming into our clinic are actually getting two meals there. It is amazing how many calories they are receiving.

Beverly Haynes: I live way out in the country, and we are in a very poor school system. When my girls were young, one of the things the school system did to offer healthier food in the elementary schools was to use local gardens. The people, the teachers, and the school employees would bring food from their gardens and supplement the cafeteria food with it, and so the school had a lot better selection. Those people have retired, and I think the schools have gone back to what is mandated. I was very pleased with the selection we had when my children were in elementary school.

Lisa Rowen: I think anything that could support local farmers and use fruits, vegetables, and produce that are grown locally so they are fresher would be wonderful for all of us in every setting.

Lori Mooney: Kudos to all of you for bringing up nutrition issues, and what you said about the school system. Some schools are doing a much better job at providing more fruits and vegetables for the kids to access as additional items or extra items that they can have. At this point, many schools are decreasing the fat intake by eliminating fried foods. They are baking things. However, there is still room for improvement, beginning with increasing better options for breakfast and lunch. Teaching kids about where food comes from and the community gardening concept is really powerful.

Beverly Haynes: That is one of the Obama incentives.

Stephenie Wallace: Returning to what the government can do, I think if the government could encourage more states and more health plans to pay for nutritional counseling, we will not be asking for more surgeries. We could start intervening at a younger age. In our center for weight management, we see infants as young as one and a half years old. I want to see them that young, because I have the most hope that by putting a fire under the adults to make some changes at that young age, that when the children are 16 they will not be in a position where they need surgery to save their lives. But the only way to do that is frequent follow-up with appropriate people that can counsel in their area of expertise.

Meg Zeller: I agree with what you were just saying. If you think about the current generation of adolescents, who will move into young adulthood and eventually have children, if they have not had success at weight loss, regardless of what they tried, they will be an extremely obese young adult or adult having children, and then those children have the cards already dealt to them for being at high risk for being obese themselves. I agree that we need to be 
focusing on the current generation, but it is the next generation, given the prevalence of obese teens who are going to mature, with whom we have a lot of work to do on the prevention side.

Lisa Rowen: I think you have made some great points in those comments. I would like to shift us a little bit at this point to talk about bariatric surgery. What do you believe recent research has revealed about indicators for selection criteria related to bariatric surgery for adolescents?

Meg Zeller: At this point, I think we have descriptive research that talks about initial safety and short-term efficacy. What we do not have, because it requires large samples, are data that would indicate who is a good patient or who is the best patient. I think we are going to be a decade into it until we really know these types of indicators. There are certainly criteria if a person has certain medical comorbidities or a certain BMI that makes them appear to be a better candidate because of risk and need. But I wish we had some type of psychosocial algorithm that would tell us what the best indicators are for good outcomes.

Beverly Haynes: I agree completely. We do not have good indicators right now. What we are using is fair, and it is better than nothing. I know that you see this problem in Cincinnati, too. It is true as well that we make our best assessments and then realize that some of these parents and patients have pulled the wool over our eyes. They know exactly what to say to us to get us to accept them and move forward with the surgery. Then it is just disastrous. It really breaks my heart, and it just makes me wonder what we can do and how soon we can get a better algorithm for getting these kids, and their families, properly assessed. I think if we could just do a "parentectomy" on them, the situation would improve.

Stephenie Wallace: I think it goes back to what we were saying in the beginning about the role of family and parents caring for our teens, even with our bariatric teens. Our pre-surgery visits should allow the families to determine if this is the right choice for their teen. It is easy for them to adopt bad habits (e.g., drinking and too many carbs) and if they do so, it could be harmful. If a family and teen do not think they can comply, then the surgery is not for them.

Lisa Rowen: Let me flip the question that I asked you earlier about "Has research supported any particular indicators for selection criteria?" to the question "Have you established some criteria where you can firmly rule out people for bariatric surgery or bariatric procedures, weight loss procedures that require close clinical follow-up for safety and efficacy?"

Stephenie Wallace: Do you mean criteria that we have developed through research that demonstrates who should not have bariatric surgery?

Lisa Rowen: I think this is interesting. In listening to your comments and Meg in particular, when you said that you think it is maybe ten years out that we will have research that supports useable criteria. Are we functioning sort of intuitively at this time? Are we "intuitively" ruling out different families, children, and adolescents for weight loss procedures? I am just trying to understand. If these procedures or programs are not very clearly indicated, if our decisions are not based so much on research, then are you using your best clinical judgment and possibly intuition? I am just wondering if we are functioning more by ruling people out, since we cannot say definitively what the indicators are for selection.

Lori Mooney: Remember, these are adolescents that we are talking about, not younger children. I want to make sure that that is understood, that these are adolescents undergoing bariatric surgery.

I think the other thing to remember is that all these related issues really do require a unique approach, for both nutritional intervention and behavioral issues. We do use the adult model to start with; however, we realize that these young people are undergoing significant changes as they grow through their adolescence. They are going through changes that adults have already experienced. We are hoping to attack these issues early on with the adolescent so we can eliminate some of the unpleasant encounters that overweight adults experienced. With that being said, there are red flags that you see in families when they are just 
not prepared to move forward in a positive manner. While these behaviors need to be addressed, they are, unfortunately, not something on which you can move quickly.

Beverly Haynes: Lori, you were speaking about the adult model. I know that our criteria are based on a model that was set up by pediatric surgeons, one that the adult world has kind of adopted. I guess I was a bit confused about which criteria we were discussing.

Lori Mooney: Let me clarify. Originally, when we were looking at all of these issues, we were using an adult approach and trying to standardize it for the adolescents, changing the program to deal with all the issues specific to this patient population. We are now doing it at a pediatric surgery level and integrating nutritional interventions.

Lisa Rowen: Let me ask if we have seen any particular surgical procedures which have better outcomes for adolescents than for adults. As well, are there any particular surgical procedures that simply have better outcomes than other surgical procedures?

Meg Zeller: I'll address that, Lisa. Laparoscopic adjustable gastric banding is not approved yet for adolescents younger than 18 in the United States. The initial data from the ongoing clinical trial have yet to be released for the FDA to consider approval. So it is hard to say. However, the data coming out of Australia about the laparoscopic gastric adjustable banding in adolescents is promising and was recently published in JAMA. Gastric bypass remains the most commonly performed procedure in adults and in teens. The sleeve gastrectomy is actually on the increase in terms of use in adolescents, but again, there is not much data out there.

Lori Mooney: We are currently involved in a clinical trial. We are halfway through a five-year study, so we do not have the data at this point. I am told we may have some published data this summer to work with or early in the fall, and I am hoping that it shows efficacy for Lap-Band. We are just one site of several doing Lap-Band. We are starting to see an increase in the sleeves at this point in time, and we are seeing potentially fewer issues with malabsorption with the Roux-en-Y. You certainly have to take a look at iron levels and $\mathrm{B}_{12}$ and all kinds of malabsorption issues that can happen with the traditional Roux-en-Y. But remember, to date, the Roux-en$\mathrm{Y}$ is still the procedure that is most frequently performed in the United States.

Lisa Rowen: Thank you for those interesting comments on technique and where we stand. I'd like to talk a bit about the quality of life before and after a surgical weight loss procedure. Are there any thoughts?

Meg Zeller: As you know, what I do day in and day out is study the quality of life of our patients. I think all of you will agree that teens who present for surgery experience significant impairment in quality of life. The data certainly supports that some of the worst quality of life has been documented in the pediatric obesity literature. It is actually alarming. And the quality of life issues are palpable to them, one of the major comorbidities with which they present. Girls know they have PCOS because someone told them, and it is difficult because they have some symptoms, but their quality of life is really what they can talk to you about most clearly.

We know the initial data, whether it is from the United States or other countries, suggest that quality of life dramatically improves following surgery, even if they remain extremely obese. Our early published data, for example, from Cincinnati, shows our mean BMI as 59, with the highest individual BMI in the sample being 88 pre-surgery. So, they are coming in at a high BMI, and they are only going to lose a certain percent of their excess weight. We are learning you can only lose so much through the procedure, and they are ending up remaining obese, if not extremely obese. Yet they have this dramatic improvement in quality of life, which I think is impressive and very interesting, too.

This begs the questions "Is the quality of life improvement about just the fact that they have had a change in their weight status versus their resulting weight?" And "Can they have an improved life, even remaining extremely obese, because it is where they came from as opposed to where they ended?" 
Stephenie Wallace: I find what Dr. Zeller is saying very interesting. I can speak anecdotally about several of our young people that we follow pre- and post-surgery in our clinic, and I definitely agree. I can speak particularly of several of our young African American female patients who have had bariatric surgery. Although they have not lost the ideal amount of weight that we had expected at their respective time points since their surgeries, when you talk to them they are glowing. They are extremely satisfied with the amount of weight they have lost. We, as a team, are still trying to motivate them to keep striving to lose weight. We try to motivate them to get the most benefit from having the surgery even though they feel wonderful about their body image and the way that the world is reacting to their smaller weight at this point.

Also, I don't want to minimize their progress because I feel like they have benefited socially from the weight they have already lost. They are interacting more with the world and dating and hanging with their friends. Our team is having more conversations with them about how their life has changed after the weight loss. For example, dating becomes more important, because many of our adolescents may not have had the "how to date" conversation beforehand; they had withdrawn from the world. But after surgery they are doing so much more, it is a wonderful thing to see.

Lori Mooney: It is a wonderful thing to see. These are kids that are losing $60 \%$ to $70 \%$ of their weight, not the total weight loss they may have anticipated initially.

Beverly Haynes: I agree. There are a couple of kids that we have had in our program who have become completely normal as far as their BMI goes, and it has just really revolutionized their lives. Their current weight and outlook seem to be maintained. I hope that that is going to be true throughout, but they seem to be holding on to that improved quality of life.

Stephenie Wallace: I will just add that I think a lot of our young people see their futures very differently after surgery. There are things they believe they can accomplish, things that they never thought they could accomplish before. I think that is really one of the powerful aspects about teenagers and working with them, being able to encourage them to reach their full potential. I have enjoyed that part of the experience.

Lisa Rowen: I would like to touch on two more topics. The first one is if you could discuss some of the ethical issues that you believe may be related to surgical weight loss procedures for adolescents.

Stephenie Wallace: One of the things that we always debated in our center when it comes to bariatric surgery is who has guardianship. There may be cases where the parent does not have legal guardianship but still plays an active role in the teen's life. It is important to allow that parent to ask questions and voice concerns to us pre- and post-operatively about this lifechanging procedure. We try to clarify everyone's role, whether a parent or legal guardian, to ensure that all have the opportunity to express themselves.

Beverly Haynes: Another issue that we struggle with when we get together as a group to discuss our bariatric surgery patients is their age. Is a 13-year-old child really ready to make this decision? I will base the decision on physical problems and their enthusiasm and the parents' support. We still really struggle with whether this is the absolute, correct thing to be doing on a young teen. So that is a concern.

Lisa Rowen: Thank you. One last question, and that is "Would you describe the barriers to surgical referral for adolescents with extreme obesity as you see them?" We see barriers in adults, with adult referrals. So I was wondering if you have any insight related to referrals for adolescents, and what can we do to address the barriers? You may have identified one already, related to age. It may be that primary care physicians are uncomfortable regarding children of very young ages, but you can speak about that or any other thoughts you may have related to barriers to referral.

Lori Mooney: Ongoing education for our insurance companies on the necessity of bariatric procedures for adolescents is imperative. We need to address behavioral and medical changes that can follow as a result. Fortunately, or 
unfortunately, we continue to have dilemmas with insurance companies in terms of coverage, and for some families, it comes down to that.

Beverly Haynes: I have to agree with Lori and add the fact that some of the pediatricians that make the referral have to be begged by the families to be referred to a weight loss center. The families tell me that their doctors will say that it is just a matter of getting up and pushing away from the table, and the kids do not really need any help. We are still combating some of the old-school thoughts.

Stephenie Wallace: I am going to add the importance of resources to properly evaluate all the families and potential teens for surgery that are coming into the center. It takes a team of 6-7 different disciplines (surgeon, pediatrician, nursing, social work, dietician, psychology, physical therapy/exercise physiologist), which requires a lot of clinician time, clinic time, and space. Recognizing the teens interested in surgery are children, so to do a thorough job in the evaluation and preparation of them for bariatric surgery, these elements are vital in doing so. We want to give them as much support from all of the disciplines before and after the surgery, so we will need more resources to do so. Adults having the surgery may not need as much.

Lori Mooney: The only other thing I would say is that there is a lack of data on the predictive factors for success in pediatric patients undergoing any kind of bariatric surgery. I think the other thing we have to remember, as we educate pediatricians, insurance companies, and all who are involved peripherally with our pa- tients, is that we can take a look at resolution or improvement with certain comorbidities. This may help us define the success of any kind of bariatric procedure. So, if we resolve their type 2 diabetes and the hyperlipidemia and the hypertension, and sleep apnea, which we know are really positive indicators-this all helps in terms of selling stakeholders on this procedure.

Lisa Rowen: I want to ask if there is anything that we have not yet touched on that is relevant to the topic.

Lori Mooney: Pediatric obesity is multifaceted, and it is multifactorial, and treatment and management involve a lot of people in the healthcare community to make it happen. It is behavioral. It is nutritional. It is parenting skills. It is the surgeon. It is the physician. It entails a great many things, along with the changes that need to be made.

Meg Zeller: Perhaps salient to the audience for this particular journal, I think we need to be forward thinking about adults who are currently having bariatric surgery and who have children in their home. What does food look like in their house? How can they be agents of change so that their children, as they develop, do not also move into having this obesity battle to the extent that they did?

Lisa Rowen: That is a great point, and you were actually making that point earlier, about the next generation. I believe it is a great comment on which to close. I would like to thank you all for participating in our roundtable on pediatric obesity and bariatric surgery. 